\title{
Thermodynamic, crystallographic, and dielectric study of the nature of glass transitions in cyclo-octanol
}

\author{
Ricardo Puertas, Maria A. Rute, Josep Salud, and David O. López* \\ Laboratori de Caracterització de Materials (LCM), Departamento de Física i Enginyeria Nuclear, \\ E.T.S.E.I.B. Universitat Politècnica de Catalunya. Diagonal, 64708028 Barcelona, Spain
}

Sergio Diez

Departamento de Física Aplicada II, Facultad de Ciencias, Universidad del País Vasco. Apartado 644, E-48080 Bilbao, Spain

J. Kees van Miltenburg

Chemical Thermodynamics Group, Debye Institute, Faculty of Chemistry, Utrecht University, Padualaan 8, NL-3548 CH Utrecht, The Netherlands

\author{
Luis C. Pardo, Josep Ll. Tamarit, and Maria Barrio \\ Laboratori de Caracterització de Materials (LCM), Departamento de Física i Enginyeria Nuclear, \\ E.T.S.E.I.B. Universitat Politècnica de Catalunya. Diagonal, 64708028 Barcelona, Spain
}

Miguel A. Pérez-Jubindo and Maria R. de la Fuente

Departamento de Física Aplicada II, Facultad de Ciencias, Universidad del País Vasco. Apartado 644, E-48080 Bilbao, Spain

(Received 20 October 2003; revised manuscript received 13 January 2004; published 15 June 2004)

\begin{abstract}
The stable solid polymorphism of cyclooctanol $\left(\mathrm{C}_{8} \mathrm{H}_{16} \mathrm{O}\right.$, for short $\left.\mathrm{C}_{8}-\mathrm{OH}\right)$ is revealed to be a complex problem and only two stable solid phases, denoted on cooling from the liquid as phases I and II, are found using static (thermodynamic and x-ray diffraction) as well as dynamic (dielectric spectroscopy) experimental techniques. Both solid phases are known to exhibit glass transitions if they are cooled down fast enough to prevent transition to ordered crystalline states. Although glass transitions corresponding to both phases had been well documented by means of specific heat measurements, x-ray measurements constitute, as far as we know, the first evidence from the structural point of view. In addition, a great amount of dielectric works devoted to phase I and its glass transition, were published in the past but next to nothing relating to the dielectric properties of phase II and its glass transition. The nature of the disorder of phase II will be discussed.
\end{abstract}

DOI: 10.1103/PhysRevB.69.224202 PACS number(s): 61.43. $-\mathrm{j}$, 64.70.Pf, 65.60. $+\mathrm{a}$, 77.22. $-\mathrm{d}$

\section{INTRODUCTION}

The physics relating to the glass transition is an old and unresolved problem of condensed matter physics for which no microscopic theory exists. When a liquid is cooled fast enough to prevent crystallization, it could result in a supercooled metastable liquid in which the material retains its liquid structure. Subsequent cooling ultimately forces the system to freeze-in a disordered state to become a nonequilibrium state in a thermodynamic sense ${ }^{1}$ (the nonergodic state of the ergodic supercooled liquid; it should be noticed that a nonergodic state is related to a system for which some property is time-dependent), often referred to as structural glass $(S G)$ or liquid glass. ${ }^{2,3}$ It must be taken into account that it is almost impossible to distinguish a structural glass from a liquid through static properties alone-both being characterized by the absence of long-range structural order. Nevertheless, their dynamics are quite different and considerable experimental advances have been recently undertaken. $^{4-9}$ The glass transition is usually identified by the viscosity attaining $10^{13}$ Poise, by a jump of specific heat or, from a dynamic point of view, by a non-Debye $\alpha$-relaxation (related to the flip-flop of the molecules) with a characteristic relaxation time $\tau$ as slow as $100 \mathrm{~s}$.

In 1938 Timmermans ${ }^{10}$ described a kind of molecular ma- terials for which distinguishable reorientational motions of the molecules in solid crystalline state could occur. The molecules forming such materials are usually more or less globular in shape, providing little steric hindrance for reorientationl freedom. These compounds were denoted as plastic crystals (this name derives from Michils ${ }^{11}$ who observed that these materials can be extruded by quite small pressures) although maybe, the most appropriate name is Orientational Disordered Crystals (ODIC for short) and the solid state or phase which presents this orientational disorder can be denoted as an OD-state. In such a state, the centers of mass of the molecules form a regular crystalline lattice but the molecules are dynamically disordered in orientation. In fact ODIC-materials can present one or more OD-states at temperatures lower than their melting point. If an OD-state is cooled fast enough to prevent phase transformations to the ordered crystalline state, it could result in a supercooled metastable OD-state in which the material retains the characteristic orientational disorder and the regular lattice of the stable OD-state. In the same way as for structural glasses, subsequent cooling ultimately forces the system to freeze-in an orientational disordered state as well as crystalline state which was referred to as a glassy state ${ }^{12}$ or as an orientational glass $(O G)^{13}$ since only the orientational degrees of freedom are frozen-in. It should be noticed that OG-state is 
out-of-equilibrium, i.e., it is the nonergodic state of the ergodic supercooled OD-state. From a dynamic point of view, it seems to be that the dielectric spectra are dominated by the $\alpha$-relaxation process like in structural glasses. However, the information up to now is sparse and needs, in some cases, to be reinterpreted. In a similar way as for structural glass transitions, the orientational glass transitions can be identified by a jump of the specific heat and by a non-Debye $\alpha$-relaxation with a characteristic relaxation time $\tau$ as slow as $100 \mathrm{~s}$.

It is well-known that the molecular materials can display other kinds of disorder aside from the orientational disorder cited above. In particular, there exists one kind of molecular material for which the molecules become flexible when temperature increases in the solid crystalline state, changing dynamically their molecular conformations. These materials were denoted a Condis crystals ${ }^{14,15}$ and the solid state or phase in which only this disorder occurs was named the condis state. In such a case, would it be possible to freeze-in the conformational disorder of a condis state to yield the corresponding nonergodic state? If this is possible, it would also be logic to denote this nonergodic state as conformational glass $(C G)$ since only the conformational degrees of freedom would be frozen-in. Going much farther away, could the conformational glass transition be also characterized by a jump of the specific heat? And, from a dynamic point of view, what kind of relaxation could be characteristic of this conformational disorder? These are the basic questions that will be addressed within.

Cyclo-octanol $\left(\mathrm{C}_{8} \mathrm{H}_{16} \mathrm{O}\right.$, for short $\left.\mathrm{C}_{8}-\mathrm{OH}\right)$ attracted our interest because its flexible molecular ring-skeleton gives rise to different molecular conformations as well as to a complex solid-state polymorphism that leads to several disordered phases which could be frozen-in to yield glassy states. Many works have been published up to now relating to, its stable polymorphism, ${ }^{16-19}$ the dynamic behavior ${ }^{17,20-29}$ of the different solid states, and, surprisingly, next to nothing relating to the structural features of the solid states. ${ }^{30,31}$ As for its stable polymorphism, three solid phases, denoted on cooling from the liquid, as phases I, II, and III were reported. An exception is made of phase III; there exists unanimity with respect to phases I and II, the transition temperatures and enthalpy changes being more or less comparable. On the other hand, phase III was only obtained by Andersson and co-workers ${ }^{17,19}$ and its existence was inferred by Dworkin et $a .^{20}$ but never confirmed. As far as the nature of phase I is concerned, it was reported as orientationally disordered (OD) from dielectric ${ }^{17,21-29,31}$ as well as $\mathrm{NMR}^{22}$ measurements. As for the nature of phase II, Dworkin et al. ${ }^{20}$ argued that there exists some kind of disorder. Leslie-Pelecky et al. ${ }^{26}$ said about phase II, on the basis of earlier works, ${ }^{17,19,23}$ that it is OD but with molecular reorientations limited to those about an axis with nearly zero dipole moment, making the dielectric susceptibility of this phase very small.

Thermal evidences of the existence of two glass transitions in $\mathrm{C}_{8}-\mathrm{OH}$, associated to phases I and II, were reported in the past. ${ }^{18,20}$ In Refs. 25 up to 29 , the dielectric loss spectra were exhaustively studied in both the stable and the supercooled phase I and even in its nonergodic state. In the present work we report on thermodynamic, crystallographic, and dielectric results concerning the glass transitions associated to these two phases. As for the thermodynamic study, the specific heat data in the stable, supercooled, and glassy state of phases I and II were obtained through Modulated Differential Scanning Calorimetry (MDSC) as well as Adiabatic Calorimetry (AdC) techniques. As far as the crystallographic study is concerned, a detailed $\mathrm{x}$-ray investigation enables us to follow the lattice symmetry of both phase I and phase II, when the sample is cooled down to their respective glassy states. The dielectric study was made in phase I as well as in phase II in order to get information about the disorder in both phases. Compared to the earlier publications, ${ }^{25-29}$ we provide additional data in the temperature gap 205-240 K for the supercooled phase I and, the most important, new data relating to the primary $\beta$-like process in phase II, which is followed for the whole temperature range of existence as a stable or possibly supercooled state. In particular, these data allow us to get information about what kind of disorder exhibits phase II and also about the nature of the glass transition associated with this phase.

The paper is organized as follows. In Sec. II we describe the experimental details. In Sec. III, the thermodynamic, crystallographic, and dielectric results related to the ergodic and nonergodic states of phases I and II are presented. In Sec. IV, a discussion is performed and finally, in Sec. V, we summarize the main conclusions.

\section{EXPERIMENTAL DETAILS}

\section{A. Material}

Cyclo-octanol was obtained from Across Organics with purity higher than $99 \%$ and was purified by means of several sublimation and crystallization processes. The compound was always handled with extreme caution in an Ar controlled atmosphere.

\section{B. Thermal measurements}

The specific-heat data were obtained by means of MDSC and AdC techniques. As for the former, a commercial TA Instruments DSC 2920 equipped with a cryogenic cooling accessory $(123 \mathrm{~K})$ was used. The MDSC technique enables us to perform measurements as a conventional DSC or in a modulated mode. In such a mode, the conventional temperature program (linear-time temperature program) is superimposed with a periodic temperature change (usually sinusoidal) which constitutes the perturbation. The response is embodied in the heat flow rate that, by means of an appropriate mathematical treatment, can be separated in two components: a dynamic component arising from the temperature modulation and the static component due to the conventional temperature program. Under suitable conditions (linear response and steady state), the specific heat data can be directly obtained in a single experiment. More details on the MDSC technique can be found somewhere else. ${ }^{32-35}$ The MDSC measurements were made on cooling and on heating at scanning rates of $2 \mathrm{~K} \cdot \mathrm{min}^{-1}$. The quenching rate in some cases was $20 \mathrm{~K} \cdot \mathrm{min}^{-1}$. The modulated temperature amplitude and modulation period were set equal to $\pm 0.5 \mathrm{~K}$ and 60 
$\mathrm{s}$, respectively. Likewise, the sample masses were selected to ensure a uniform thin layer within the aluminum pan. The specific heat calibration was performed using pure synthetic sapphire, although the absolute accuracy is usually no better than 5 percent or even worse.

As for the specific heat obtained by means of the AdCtechnique, a home-made adiabatic calorimeter (the name of the designation in the Chemical Thermodynamics Group Laboratory is CAL V) described elsewhere ${ }^{36,37}$ was used. The vessel was filled with a mass of about $10 \mathrm{~g}$ in a controlled atmosphere of nitrogen gas. After being filled, the vessel was evacuated and filled with about $1000 \mathrm{~Pa}$ of gas helium in order to improve heat conduction. Measurements were performed in the so-called intermittent mode, for which stabilization periods at each temperature of about $600 \mathrm{~s}$ were used between energy input periods of about $800 \mathrm{~s}$. The reproducibility of the calorimeter is about 0.01 per cent and was checked with standard materials as synthetic sapphire and $n$-heptane showing no deviations larger than 0.2 per cent from recommended values. The maximum quenching rate was $5 \mathrm{~K} \cdot \mathrm{min}^{-1}$.

\section{X-ray powder diffraction measurements}

$\mathrm{X}$-ray powder diffraction data were collected using a horizontally mounted INEL cylindrical position-sensitive detector (CPS-120) equipped with a liquid nitrogen INEL CRY950 cryostat $(80-500 \mathrm{~K})$. The detector, used in Debye-Sherrer geometry, consists of 4096 channels and the angular step was $0.029^{\circ}(2 \theta)$. Monochromatic $\mathrm{Cu} \mathrm{K} \alpha_{1}$ radiation was selected. The samples were introduced into $0.5 \mathrm{~mm}$ diameter Lindemann glass capillaries and were rotated around the $\theta$ axis during the experiments. External calibration using the cubic phase $\mathrm{Na}_{2} \mathrm{Ca}_{3} \mathrm{Al}_{2} \mathrm{~F}_{4}$ was performed to convert channels to

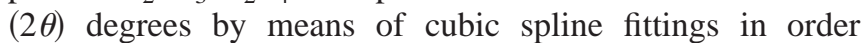
to correct the deviation from angular linearity in PSD. DIFRACTINEL software was used for the calibration and for the peak position determinations after Pseudo-Voigt fittings in the standard measurements.

\section{Dielectric measurements}

The measurements of the complex permittivity $\varepsilon^{*}(\nu)$ $=\varepsilon^{\prime}(\nu)-i \varepsilon^{\prime \prime}(\nu)$ were performed with an Alpha impedance analyzer $\left(10^{-3}-10^{6} \mathrm{~Hz}\right)$ from Novocontrol. The cell consists of two gold-plated brass electrodes (diameter $10 \mathrm{~mm}$ ) separated by two $50 \mu \mathrm{m}$ silica spacers making a plane capacitor. The sample was held in a cryostat, the temperature being controlled by a System Quatro from Novocontrol using a heated nitrogen gas stream. Additional details can be found somewhere else. ${ }^{38}$ The material was introduced into the dielectric cell in liquid state and sample capacity was always checked before the measurements. Data acquisition was made in the temperature domain ranging between $145 \mathrm{~K}$ and $293 \mathrm{~K}$. The maximum quenching rate was $15 \mathrm{~K} \cdot \mathrm{min}^{-1}$. The measurements were performed on heating and on cooling with stabilization at different temperature steps with a temperature control of $\pm 0.5 \mathrm{~K}$.

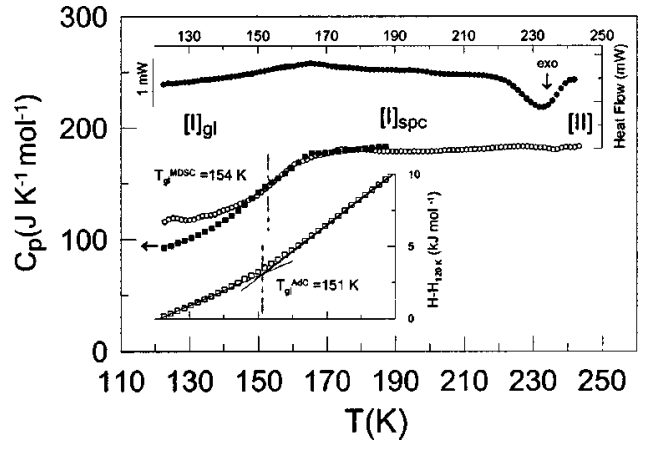

FIG. 1. Specific-heat data as a function of temperature relating to the glass transition of phase $\mathrm{I}$ of $\mathrm{C}_{8}-\mathrm{OH}$. Open circles and solid squares correspond to data from MDSC and AdC techniques, respectively. Open squares on the inset at the bottom correspond to the integrated enthalpy data from the AdC technique. Solid circles on the inset at the top, correspond to the heat flow, simultaneously recorded, using the MDSC technique. $[\mathrm{I}]_{\mathrm{gl}}$ stands for the glassy state of phase I. $[\mathrm{I}]_{\mathrm{spc}}$ stands for the supercooled state of phase I.

\section{RESULTS}

\section{A. Thermodynamic study}

As it has been recently referred, ${ }^{31}$ only two stable solid phases at normal pressure have been observed for $\mathrm{C}_{8}-\mathrm{OH}$ (denoted on cooling from the liquid state as phases I and II). An additional stable phase at normal pressure induced from high-pressure measurements, denoted as phase III, was reported in the past by other authors, ${ }^{17,19}$ but no further confirmation was obtained.

Phase I was earlier reported as an OD-phase ${ }^{19,22,29}$ and it is well-known that it can be super-cooled by means of a more or less rapid quench giving rise (if the quench is deep enough) to an OG. Figure 1 displays the specific heat data obtained on heating from glassy state of phase I (denoted in the figure as $[\mathrm{I}]_{\mathrm{gl}}$ ) using the MDSC technique together with that obtained by means of adiabatic calorimetry. The specific heat jump (or anomaly) as a function of temperature characterizing the typical relaxation process from the glassy state to the super-cooled phase $\mathrm{I}\left([\mathrm{I}]_{\mathrm{spc}}\right)$ can be clearly observed. At the bottom of Fig. 1, as an inset, the integrated enthalpy was calculated from the AdC-data, according to the following expression:

$$
H_{T}=H_{T_{0}}+\int_{T_{0}}^{T} C_{p} d T
$$

in which $T_{0}$ is in this case $120 \mathrm{~K}$. From this inset, the glass transition temperature is read to be $151 \mathrm{~K}$. In Table I, the glass transition temperatures of the glassy state I, obtained from both experimental techniques, MDSC and AdC, are presented together with those referenced in the literature. It is important to realize that in the OG-state, the absolute value of the specific heat data obtained from both experimental techniques is slightly different. This is not surprising because of the different time-scaling in measuring the specific heat in a nonequilibrium state. Nevertheless, it may be underlined that both glass transition temperatures are virtually identical despite the time-scaling. 
TABLE I. Literature sources for the glass transition temperatures of phase I and II of $\mathrm{C}_{8}-\mathrm{OH}$ along with those determined in this work.

\begin{tabular}{cccc}
\hline \hline$T_{[\mathrm{I}] \mathrm{gl}}(\mathrm{K})$ & $T_{[\mathrm{II}] \mathrm{gl}}(\mathrm{K})$ & Experimental technique & References \\
\hline $162^{\mathrm{a}}$ & $143^{\mathrm{a}}$ & Adiabatic calorimetry & 18 \\
142 & 132 & Differential thermal analysis & 20 \\
155 & $\ldots$ & Dielectric spectroscopy & 21 \\
150 & $\ldots$ & Thermally stimulated current, & 22 \\
& & nuclear magnetic resonance & 26 \\
165 & $\ldots$ & Dielectric spectroscopy & 27 \\
168 & & Dielectric spectroscopy & 29 \\
164 & $\ldots$ & Differential scanning calorimetry & \\
172 & $\ldots$ & Dielectric spectroscopy & This work \\
151 & 134 & X-ray diffraction & \\
164 & 135 & Dielectric spectroscopy & \\
154 & 144 & Adiabatic calorimetry & \\
151 & 135 & Modulated differential scanning calorimetry & \\
\hline \hline
\end{tabular}

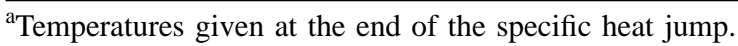

${ }^{b}$ DSC measurements at $10 \mathrm{~K} \mathrm{~min}^{-1}$ and annealed at $100 \mathrm{~K}$ for a time of $10 \mathrm{~min}$.

${ }^{\mathrm{c}}$ Temperatures according to the procedure and to the specified conditions in this work.

At the top of Fig. 1, also as an inset, the MDSC heat flow which is simultaneously recorded with the specific heat data is also plotted. The exothermic peak around $230 \mathrm{~K}$ corresponds to the transition between the supercooled phase I and the phase II which is presumably stable at this temperature. If an appropriate cooling (at about $2 \mathrm{~K} \cdot \mathrm{min}^{-1}$ or even slower), from one temperature just above the exothermic peak shown in Fig. 1 (the sample being in phase II) is performed down to $100 \mathrm{~K}$, an interesting behavior is making clear on the successive heating from about this temperature, as it can be observed in Fig. 2. In such a figure, specific heat data on heating by means of both experimental techniques, MDSC and AdC, are shown. The MDSC heat flow is plotted at the top of the figure, as an inset. It is important to realize that in the heat flow no exothermic or endothermic peaks are observed up to $260 \mathrm{~K}$, a temperature very close to the II to I phase transition which for clarity is only shown in the MDSC specific-heat data. On the other hand, a specific heat

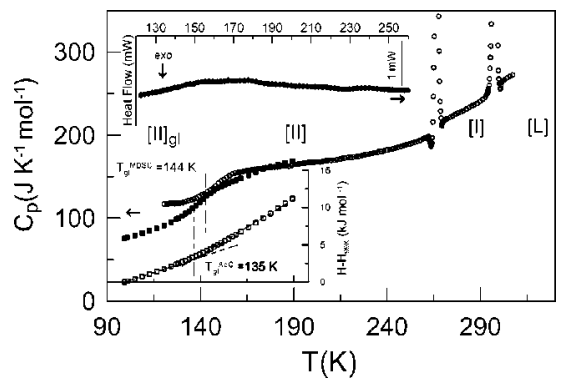

FIG. 2. Specific-heat data as a function of temperature relating to the glass transition of phase II of $\mathrm{C}_{8}-\mathrm{OH}$. Open circles and solid squares correspond to data from MDSC and AdC techniques, respectively. Open squares on the inset at the bottom, correspond to the integrated enthalpy data from the AdC technique. Solid circles on the inset at the top, correspond to the heat flow, simultaneously recorded, using the MDSC technique. $[\mathrm{II}]_{\mathrm{gl}}$ stands for the glassy state of phase II. anomaly or jump is observed irrespective of the technique at the low-temperature side. As it has been cited, this behavior in the specific-heat evolution with temperature is representative of a relaxation process associated to a glass transition. In the present case, the glass transition should be related to the existence of the nonergodic state of the phase II, denoted in Fig. 2 as $[\mathrm{II}]_{\mathrm{gl}}$. This possibility was argued by Dworkin et $a .^{20}$ and much more recently by Sciensinsky et al. ${ }^{18}$ In Table I, the glass transition temperatures related to the glassy state II obtained from both the MDSC and AdC techniques are consigned together with those previously published. Again, the AdC glass transition temperature is obtained from the integrated enthalpy values depicted at the bottom of Fig. 2 , as an inset. It should be noticed that, in such a case, the difference between the AdC and MDSC glass transition temperatures is almost ten degrees. Likewise, the differences in the absolute value of the specific-heat data reported for the nonergodic state II by means of both experimental techniques is also evident as in the preceding case (the nonergodic state I).

\section{B. Crystallographic study}

Preliminary $\mathrm{x}$-ray powder diffraction experiments on $\mathrm{C}_{8}-\mathrm{OH}^{31}$ revealed that phase $\mathrm{I}$ is simple cubic with a unitcell parameter of $11.96(1) \AA$ at $273 \mathrm{~K}(Z=8$; eight molecules per unit cell) contrary to the result previously reported $^{30}$ as face centered cubic symmetry ( $a=9.56 \AA$ with $Z=4)$. After a fast $\left(5-10 \mathrm{~K} \cdot \mathrm{min}^{-1}\right)$ and deep enough quench of the sample in phase I, the X-ray profiles were recorded at different temperatures every $10 \mathrm{~K}$ step in the temperature range between $93 \mathrm{~K}$ and $293 \mathrm{~K}$. Because phase I can be easily supercooled to very low temperatures, the variation of the molar volume $(v)$ against temperature can be determined (Fig. 3). A continuous variation of the molar volume against temperature as well as a change in the slope at one temperature are signatures of a glass transition between 


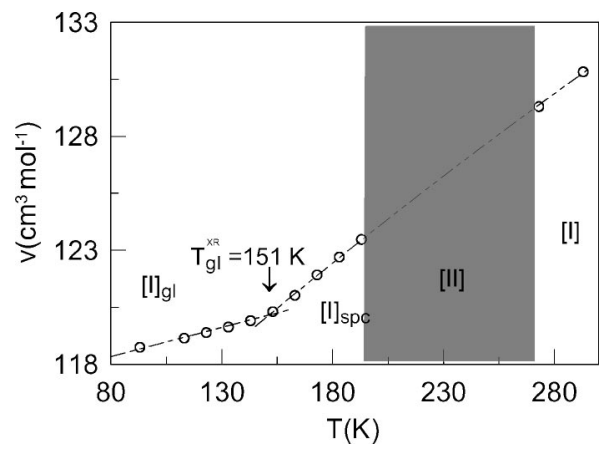

FIG. 3. Molar volume for phase $\mathrm{I}$ of $\mathrm{C}_{8}-\mathrm{OH}$ against temperature derived from $\mathrm{x}$-ray diffraction experiments.

$[\mathrm{I}]_{\mathrm{gl}}$ and $[\mathrm{I}]_{\mathrm{spc}}$, the temperature of which is consigned in Table I. It may be underlined that this temperature is very close to that determined using MDSC and AdC techniques.

For phase II, the characteristic x-ray powder diffraction profile (which was assigned to phase II in a preceding work $^{31}$ ) is found to be present down to $100 \mathrm{~K}$ from about 240 $\mathrm{K}$ (the temperature at which phase II is formed when the sample in $[\mathrm{I}]_{\mathrm{spc}}$ is heated; see Fig. 1). Unfortunately, all attempts to index the x-ray profile of phase II have been unsuccessful, and so the symmetry of phase II remains unknown. Despite this fact, the spacing $d_{h k l}$ of unambiguously defined reflections with unknown $h k l$-Miller indices of the $\mathrm{x}$-ray diffraction profile can be followed as a function of temperature. In Fig. 4, the spacing of two of these reflections (denoted as $d_{A}$ and $d_{B}$ ) are plotted against temperature. At the top of Fig. 4, the x-ray powder patterns for a short $d$-range at 233.15 and $103.15 \mathrm{~K}$ are shown. From this inset, similar patterns with the same reflections are observed at the maximum and minimum measured temperatures. The variation of both $d_{A^{-}}$and $d_{B}$-spacings with temperature is continuous but the change in their slopes at one temperature reveals the existence of a glass transition between $[\mathrm{III}]_{\mathrm{gl}}$ and [II], the temperature being consigned in Table I. It is worth noting that this glass transition temperature is very close to that reported by means of an $\mathrm{AdC}$ technique.

\section{Dielectric study}

Figure 5 shows the real part $\varepsilon^{\prime}(\nu)$ of the complex dielectric permittivity $\varepsilon^{*}$ on cooling down to $210 \mathrm{~K}$ from the liquid state and on the subsequent heating, at $\nu=1 \mathrm{kHz}$. The measurements were made every $1 \mathrm{~K}$ both on cooling and on heating. As it can be observed, on the slow cooling regime, the liquid state transforms to phase I which remains as a supercooled state down to about $220 \mathrm{~K}$. At about this temperature, a time-dependent transition to a more ordered phase occurs. An annealing at $210 \mathrm{~K}$ for a period of time of about three hours was proven to be enough to complete the transition. A subsequent heating from $210 \mathrm{~K}$ allows us to observe a phase transition at about $265 \mathrm{~K}$ towards phase I and another one at a higher temperature in which the melting takes place. So, the phase below $265 \mathrm{~K}$ was identified as phase II according to thermal and $\mathrm{x}$-ray measurements (see Secs. III A and III B) as well as to the bibliographic sources. ${ }^{17,19,25-28,31}$

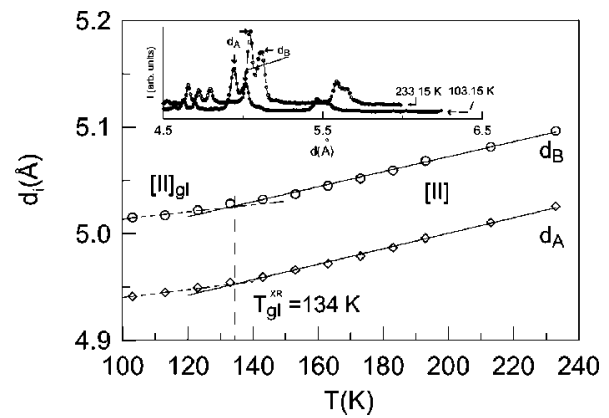

FIG. 4. Evolution of two arbitrary $d_{h k l}$ directions (denoted for simplicity as $d_{A}$ and $d_{B}$ ) of the unknown lattice of phase II of $\mathrm{C}_{8}-\mathrm{OH}$, as a function of temperature. The inset shows the $\mathrm{x}$-ray diffraction profiles at the maximum and minimum measured temperatures and the chosen $d_{A}$ and $d_{B}$ directions.

As an inset in Fig. 5, the behavior of the real part of the permittivity at around $225 \mathrm{~K}$ is shown in detail at $0.1 \mathrm{kHz}, 1$ $\mathrm{kHz}$, and $10 \mathrm{kHz}$ in order to elucidate the existence or not of a phase transition at $225 \mathrm{~K}$. It seems that in the same way as for our thermal and x-ray measurements, no evidence about phase III to phase II transition is observed. So, the dielectric results were interpreted on the basis of the existence of two stable solid phases, phase I and phase II. The complex dielectric permittivity was fitted to the phenomenological Havriliak-Negami (HN) function,

$$
\varepsilon^{*}(\nu)=\varepsilon_{\infty}+\frac{\Delta \varepsilon}{\left[1+(i 2 \pi \nu \tau)^{\alpha_{\mathrm{HN}}}\right]^{\beta_{\mathrm{HN}}}},
$$

where $\varepsilon_{\infty}$ stands for the high-frequency limit of the dielectric permittivity, $\Delta \varepsilon\left(=\varepsilon_{S}-\varepsilon_{\infty}\right.$; where $\varepsilon_{S}$ is the static permittivity) for the dielectric strength, $\alpha_{\mathrm{HN}}$ and $\beta_{\mathrm{HN}}$ determine the spectral shape, and $\tau$ is the relaxation time which is related to the frequency of maximum losses through

$$
\tau=\frac{1}{2 \pi \nu}\left[\tan \left(\frac{\pi}{2\left(\beta_{\mathrm{HN}}+1\right)}\right)\right]^{1 / \alpha_{\mathrm{HN}}} .
$$

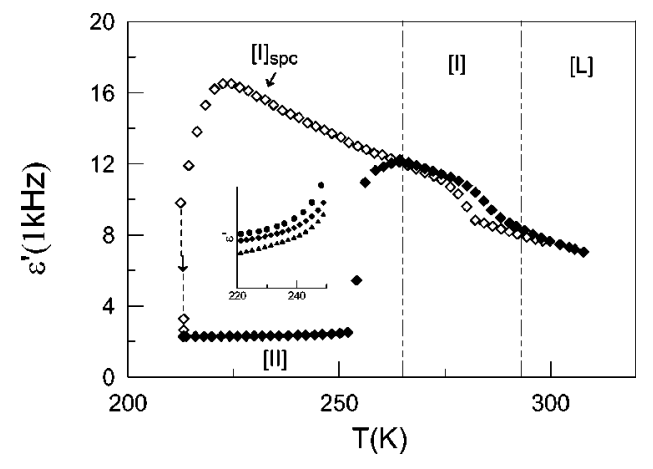

FIG. 5. Temperature dependence of the real part of the dielectric permittivity of $\mathrm{C}_{8}-\mathrm{OH}$ at $1 \mathrm{kHz}$. Open and solid diamonds stand for cooling and heating runs, respectively. The inset shows the real part of the dielectric permittivity at around $225 \mathrm{~K}$ at $0.1,1$, and $10 \mathrm{kHz}$. 


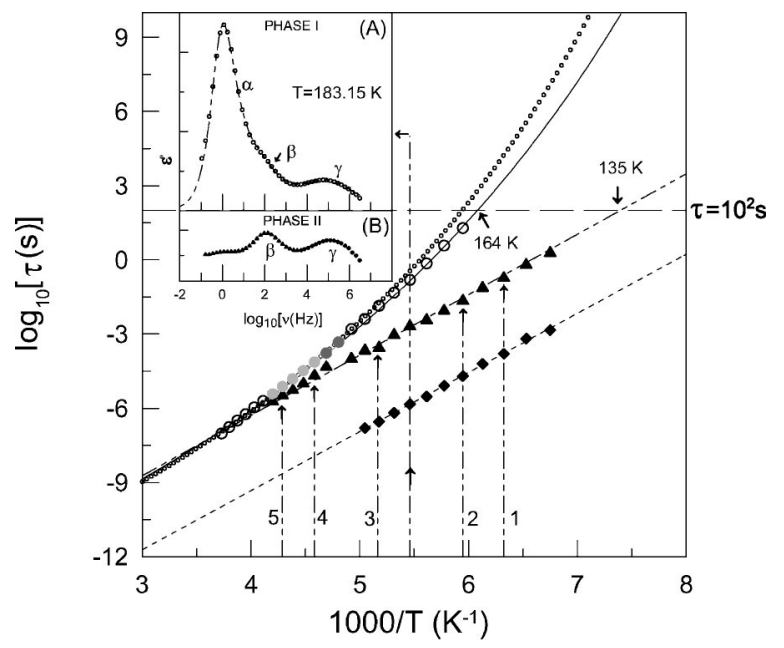

FIG. 6. Relaxation time of $\alpha$-, $\beta$ - and $\gamma$-processes in an Arrhenius plot. The solid line corresponds to the VFT-fitting of the $\alpha$-relaxation data in phase I (open circles and solid-gray circles) and the ooo-line corresponds to the Brands' VFT-fitting according to Ref. 27. The dot-dashed and dashed lines are fits of $\beta$-relaxation data (solid triangles) and $\gamma$-relaxation data (solid diamonds), respectively, both in phase II, according to Arrhenius behavior. The inset shows the typical dielectric loss spectra measured in supercooled phase I (A) and in phase II (B) at $183.15 \mathrm{~K}$

The four adjustable parameters $\varepsilon_{\infty}, \alpha_{\mathrm{HN}}, \beta_{\mathrm{HN}}$, and $\tau$ were simultaneously determined at each temperature in a fitting procedure of the $\mathrm{HN}$-function [Eq. (2)].

The dielectric loss spectra of $\mathrm{C}_{8}-\mathrm{OH}$ in phase $\mathrm{I}$, as it was reported by other authors, ${ }^{25-29}$ show a well-developed $\alpha$-relaxation peak along with two additional relaxation processes [see inset (A) of Fig. 6]. In a similar way as per Brand et al. ${ }^{27}$ the additional relaxations were denoted as $\beta$-relaxation and $\gamma$-relaxation. This nomenclature was adopted to account for the succession of the relaxations in the frequency window and not for their physical meaning. On the other hand, when the phase transition from phase I to phase II completely occurs, $\alpha$-relaxation is fully suppressed and only $\beta$ and $\gamma$ relaxations persist in phase II [see inset (B) of Fig. 6].
As for the dielectric study of phase I, our results agree well with those reported by other authors. ${ }^{25-29}$ The analysis of the $\alpha$-relaxation loss peaks at each temperature according to Eq. (2) leads to good fits with $\alpha_{\mathrm{HN}}=1$ and $\beta_{\mathrm{HN}}<1$, proving to be quite well described as Cole-Davidson (CD). The relaxation times $\tau$, shown in Fig. 6 as empty circles, were obtained from the fits as well. The behavior of $\tau$ with temperature can be described by the phenomenological VogelFulcher-Tamann (VFT) law given by

$$
\tau=\tau_{0} e^{A /\left(T-T_{0}\right)},
$$

where $\tau_{0}$ is a pre-exponential factor, $T_{0}$ is the Vogel temperature, and $A$ is a parameter with a dimension of temperature. The solid line in Fig. 6 corresponds to the fitting of our data according to a VFT-law whereas the (ooo-line) corresponds to the Brand et al. data VFT-fitting. ${ }^{27}$ Both sets of fitting parameters together with those taken from the literature are consigned in Table II. Unlike the data from Leslie-Pelecky et al. ${ }^{26}$ and Brand et al. ${ }^{27}$ additional data are provided in the gap region between 205 and $240 \mathrm{~K}$ (gray circles in Fig. 6). As it can be observed in Fig. 7(a), when the sample in phase I is slowly cooled, the supercooled phase I remains down to about $218 \mathrm{~K}$ (the loss peak of the $\alpha$-relaxation clearly diminishes below this temperature, exhibiting a slow phase transition to phase II). In fact, the gap region is now restricted to the 205-218 K temperature-region. The loss peak at $203 \mathrm{~K}$ was measured after heating the supercooled phase I which was attained by cooling the sample from phase I down to about $160 \mathrm{~K}$, fast enough to prevent the phase transition to phase II. Notwithstanding that the phase transition takes place between $205 \mathrm{~K}$ and $218 \mathrm{~K}$, data corresponding to this temperature-gap can be obtained and were included in Fig. 6 (dark-gray circles). Nevertheless, although these data line up quite-well, were excluded from the VFT-fitting procedure.

Regards the dielectric study of phase II, the loss peaks corresponding to the denoted $\beta$ and $\gamma$ relaxations [see inset (B) of Fig. 6] were recorded in a wide frequency window. The $\beta$-relaxation is followed from $145 \mathrm{~K}$ up to $240 \mathrm{~K}$, the temperature at which the $\beta$-peak exceeds the available experimental frequency window $\left(10^{6} \mathrm{~Hz}\right)$. As for $\gamma$-relaxation data, they were obtained from $145 \mathrm{~K}$ up to $190 \mathrm{~K}$. For tem-

TABLE II. Relaxation parameters of the phase I and II of $\mathrm{C}_{8}-\mathrm{OH}$ obtained in the present work and those available in the literature.

\begin{tabular}{cccccccc}
\hline \hline Phase & $\begin{array}{c}\text { Relaxation } \\
\text { type }\end{array}$ & \multicolumn{2}{c}{ Arrhenius law } & \multicolumn{2}{c}{ VFT law } \\
& $E(\mathrm{eV})$ & $\tau_{0}(\mathrm{~s})$ & $\mathrm{A}(\mathrm{K})$ & $\tau_{0}(\mathrm{~s})$ & $T_{0}(\mathrm{~K})$ & References \\
\hline I & $\alpha$ & 0.62 & $1.0 \times 10^{-18}$ & $\ldots$ & $\ldots$ & $\ldots$ & 21 \\
I & $\alpha$ & & & 2451 & $6.7 \times 10^{-14}$ & 92.4 & 26 \\
I & $\alpha$ & & $\ldots$ & 3320 & $2.0 \times 10^{-15}$ & 82.0 & 27 \\
I & $\alpha$ & $\ldots$ & $\ldots$ & 3690 & $2.6 \times 10^{-15}$ & 80.6 & 29 \\
I & $\alpha$ & $\ldots$ & $\ldots 488$ & $2.1 \times 10^{-15}$ & 73.6 & This work \\
I & $\beta$ & 0.51 & $1.8 \times 10^{-17}$ & $\ldots$ & $\ldots$ & $\ldots$ & 27 \\
I & $\beta$ & 0.49 & $3.0 \times 10^{-16}$ & $\ldots$ & $\ldots$ & $\ldots$ & 29 \\
II & $\beta$ & 0.48 & $1.1 \times 10^{-16}$ & $\ldots$ & $\ldots$ & $\ldots$ & This work \\
I & $\gamma$ & 0.47 & $2.9 \times 10^{-19}$ & $\ldots$ & $\ldots$ & $\ldots$ & 27 \\
I & $\gamma$ & 0.58 & $3.7 \times 10^{-21}$ & $\ldots$ & $\ldots$ & $\ldots$ & 29 \\
II & $\gamma$ & 0.47 & $1.3 \times 10^{-19}$ & & & & This work \\
\hline \hline
\end{tabular}




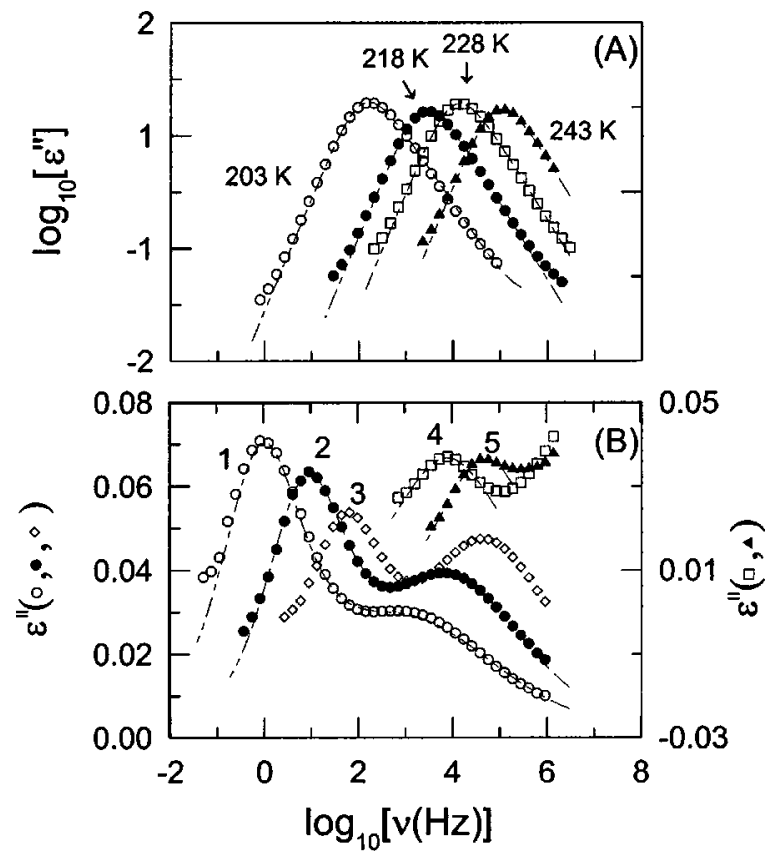

FIG. 7. Frequency dependence of the dielectric loss spectra of the $\alpha$-relaxation for various temperatures in the gap-region of phase I (A) and the $\beta$ - and $\gamma$-processes for 5 temperatures (denoted from 1 to 5 and marked in Fig. 6 by the arrows) in phase II. The dotdashed lines are fits according to the HN function [Eq. (4)].

peratures higher than $190 \mathrm{~K}$, the $\gamma$-loss peak is found at frequencies higher than $10^{6} \mathrm{~Hz}$. It is important to realize that additional measurements were performed between $10^{6}$ and $10^{9} \mathrm{~Hz}$, but all attempts to obtain reliable $\beta$ and $\gamma$ peak data were unsuccessful. Figure 7(b) shows the dielectric loss spectra of phase II for five representative temperatures which are marked in Fig. 6 as well. The 4 and 5 loss peaks, in Fig. 7 (b), correspond only to $\beta$-relaxation and were shifted up in order to better distinguish them. The dot-dashed lines are the fittings according to Eq. (2) in which a Cole-Cole (CC) behavior $\left(\alpha_{\mathrm{HN}}<1\right.$ and $\left.\beta_{\mathrm{HN}}=1\right)$ was stated for both the $\beta$ and $\gamma$ relaxations. The relaxation times $\tau$ for both relaxations in phase II, as a function of temperature are shown in Fig. 6. They clearly show a thermally activated behavior [Arrhenius law; $\left.\tau=\tau_{0} \exp (E / R T)\right]$ for which the fit parameters (energy barrier $E$ and the pre-exponential factor $\tau_{0}$ ) are consigned in Table II.

\section{DISCUSSION}

\section{A. Glassy states}

It seems doubtless from calorimetric as well as x-ray measurements that $\mathrm{C}_{8}-\mathrm{OH}$ displays two disordered solid states (phases I and II), each one with a defined kind of disorder which can be frozen-in. Nevertheless, the nature of disorder in both phases is quite different. From a structural point of view, phase I displays a cubic isotropic lattice and from a dynamic point of view a well-developed $\alpha$-relaxation is present together with two secondary relaxations, denoted $\beta$ and $\gamma$. The existence of the $\alpha$ process with high values of dielectric permittivity as well as the isotropy of the lattice are signatures of orientational disorder which can be described as being due to quasi-isotropic or endospherical reorientations (the molecules reorient around three different axes, compatible with the cubic symmetry). The thermodynamic as well as the crystallographic studies have clearly evidenced the existence of a glass transition in which the orientational disorder of phase I is frozen-in giving rise to an orientational glass $\left([\mathrm{I}]_{\mathrm{gl}}\right)$. From dielectric data, by extrapolating the relaxation time $(\tau)$ at $10^{2} \mathrm{~s}$, relating to the $\alpha$-relaxation, welldescribed by means of a VFT-law (see Fig. 6), leads to a temperature for the glass transition of about $164 \mathrm{~K}$. This temperature is about $10 \mathrm{~K}$ higher than that obtained by means of Adc, MDSC, and X-ray techniques and comparable to that obtained by other authors using dielectric spectroscopy (see Table I).

As far as phase II is concerned, from a structural point of view, the symmetry of its unit-cell is presently unknown although it seems to be lower than tetragonal. ${ }^{31}$ From Fig. 4, without doubt, phase II can be cooled down at temperatures about $140 \mathrm{~K}$ and it seems to be that at lower temperatures, ultimately phase II becomes a nonequilibrium state, i.e., nonergodic phase II because there exists a continuity in the evolution with temperature of one spacing $\left(d_{A}\right.$ or $\left.d_{B}\right)$ in the anisotropic lattice but a change in the slope of this evolution at about $134 \mathrm{~K}$ exists. From a thermodynamic point of view, when phase II is heated up from about $90 \mathrm{~K}$ a jump in the specific-heat evolution with temperature was found (see Fig. 2) at a temperature between $135 \mathrm{~K}$ (AdC-technique) and 144 $\mathrm{K}$ (MDSC-technique). It should be noted that the temperature obtained from AdC and x-ray measurements are comparable themselves and their respective time-scaling as well. It is evident that some kind of disorder that exists in phase II is frozen-in, but the following question arises: what is the nature of such a disorder? It is evident that owing to the lattice symmetry, if orientational disorder exists, it would be in such a way that the molecules would undergo jumps among distinguishable orientational positions around one axis.

The static dielectric permittivity of phase II is very low, similar to what is expected for ordered solid states. In addition, the loss spectra, irrespective of the temperature, do not display $\alpha$-relaxation and only two processes, similar to $\beta$ and $\gamma$-processes appearing in phase I, are found (see Fig. 6). So, it seems that the disorder of phase II must be dynamically described by means of these two processes that by comfort will be denoted as $\beta$ - and $\gamma$-like processes (now being primary and secondary relaxations of phase II, respectively). Moreover, this disorder inherent to phase II should be present in phase I, because these two relaxations exist in the later phase. It might be reasonably supposed that such a disorder is intra-molecular because it exists in both phases (according to the loss spectra) but very slightly modified from one phase to another (the surrounding of one molecule slightly depends on the phase) as it can be observed in Table II. It is well worth noting that the possibility of these two $\beta$ or $\gamma$-like processes accounting for some kind of orientational disorder in phase II, as it was pointed out by certain authors $^{17-19,25,26}$ some time ago, should be fully discarded. Likewise, the possibility of a dielectrically-invisible 
$\alpha$-relaxation accounting for an orientational disorder as was guessed in other works ${ }^{17,19,23,25,26}$ would seem less reasonable.

The question, now, is addressed to what kind of intramolecular dynamic disorder would be present in phase II of $\mathrm{C}_{8}-\mathrm{OH}$ ? Brand et al. ${ }^{27}$ reported some ideas about the nature of the disorder accounting for these secondary relaxations in phase I. Some of their explanations are related to side-band effects or conformational changes in which the molecules undergo transitions between the six possible threedimensional shapes of the C-octagon or transitions between the possible orientations of the hydroxyl group. We suggest that the primary process (denoted as $\beta$-like process) in phase II, could consist in one or both types of dynamic conformational disorder in which the molecule dynamically changes its conformation. The second process (denoted a $\gamma$-like process) in phase II, could be attributed to hydroxyl group rotations, much faster than the conformational changes. An argument in support of this point of view is addressed to the observed diminution of the loss curve representative of the $\beta$-like process when an electric field $(800 \mathrm{mV} / \mu \mathrm{m})$ is applied perpendicular to the electrodes-cell. According to our interpretation, the dynamic conformational disorder of the molecule is diminished because the applied field induces an alignment of the molecule limiting the conformational changes. On the contrary, under the same conditions, the loss curve representative of the $\gamma$-like process does not practically experiment significant modifications, being independent of the applied electric field as it should be expected from an internal disorder exclusively due to hydroxyl group rotations. In order to confirm our assumption about the conformational disorder of phase II, other dynamic experiments in this phase like neutron scattering or NMR should be carried out.

Now, let us consider the relaxation time $\tau$ of the primary $\beta$-like relaxation in phase II (see Fig. 6) well-described by means of an Arrhenius-law. By extrapolating this relaxation time at $\tau=10^{2} \mathrm{~s}$, the corresponding temperature would be about $135 \mathrm{~K}$, very close to that obtained by means of $\mathrm{AdC}$ and $\mathrm{x}$-ray experiments for the glass transition relating to phase II $\left([\mathrm{II}]_{\mathrm{gl}}\right)$.

It would appear, then, that if all the above considerations are correct, phase II of $\mathrm{C}_{8}-\mathrm{OH}$ should be considered as a condis state (and never as an OD state such as up to now many researchers had suggested). This condis state gives rise to a nonergodic state in which the conformational disorder is frozen-in at about $135 \mathrm{~K}$ (or up to $144 \mathrm{~K}$, depending on the experimental technique and time-scaling) and therefore a conformational glass $(C G)$ would appear.

\section{B. Fragility}

The concept of fragility was introduced in the past in order to classify the structural glass-formers. In particular, the fact that different glass-forming materials have certain similar features close to their glass transition, like the same deviation from thermally activated behavior, brought us to think that there were some aspects of the molecular disorder (those susceptible to be frozen-in) in common between them, in spite of their different chemical nature. The fragility clas-

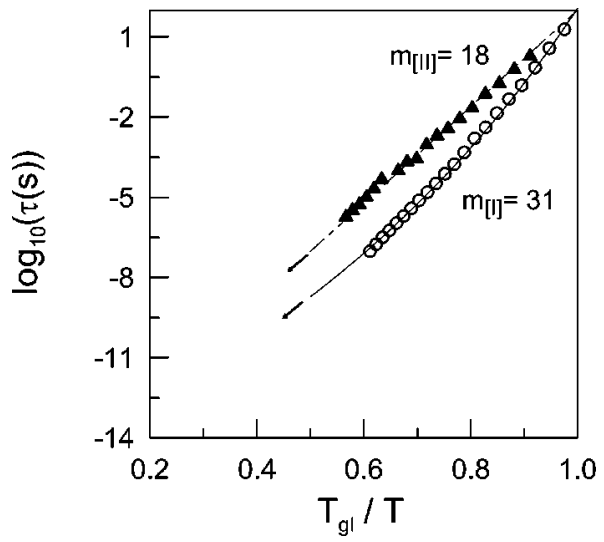

FIG. 8. Angell's plot for the $\alpha$-relaxation in phase I and for the $\beta$-relaxation in phase II. The solid and dot-dashed lines are fits according to the VFT and Arrhenius laws, respectively.

sification was earlier related to the assumption of different potential energy barriers in the configuration space. ${ }^{39}$ It is well-known for the researchers in this field that the most fragile glass-forming materials are those which show the largest deviations from thermally activated behavior. If one replaces $A$, in the VFT-law [Eq. (4)], by $D T_{0}$ (in which $D$ is the so-called strength parameter and $T_{0}$ is the already defined Vogel temperature), we can obtain a certain idea of such a deviation. The more the fragile is the material, the lesser is the strength parameter. Values of $D$ lower than 10 imply large deviations from Arrhenius law and the materials are denoted as fragile glasses.

Another basic expression that is revealed to be useful for the purpose of fragility classification is the fragility or steepness index ${ }^{40,41} m$, defined by

$$
m=\left[\frac{d \log _{10} \tau}{d\left(T_{g} / T\right)}\right]_{T=T_{g}},
$$

in which $m$ accounts for the slope at $T_{g}$ in an Angell plot $\left(\log _{10} v s T_{g} / T\right)$. The lower limit ${ }^{42}$ of $m$-fragility is assigned to 16 , the strongest materials. On the other hand, the most fragile glass-forming materials have values of $m$ up to 200 or even more. According to this way in which fragility has been defined, the $\tau$ data contained in Fig. 6, relating to the $\alpha$-relaxation of phase I, can be redrawn, as is shown in Fig. 8 (Angell's plot). The calculated value of $m$ is 31 for phase I (denoted as $m_{[\mathrm{I}]}$ for short). The $m_{[\mathrm{I}]}$ corresponds to the $m$-fragility of $\mathrm{C}_{8}-\mathrm{OH}$ as an OG-forming material, its value being comparable to that reported in the literature $\left(m_{[\mathrm{I}]}\right.$ $=33) .{ }^{25-27}$ Likewise, the strength parameter $D_{[\mathrm{I}]}$ can be calculated from the data in Table II giving rise to a value of about 47 , pointing out a relatively strong OG-forming material. Both values $m_{[\mathrm{I}]}$ and $D_{[\mathrm{I}]}$ are consistent with the relationship $m=16+(590 / D)$ deduced by Böhmer et al. 10 years ago. $^{42}$

The question, now, is addressed to the dynamic disorder of phase II in such a way that it is possible to extent the concept of $m$-fragility to a primary $\beta$-like process with an intra-molecular origin? Regardless of whether the reply is affirmative or not, the $\tau$-data contained in Fig. 6 , relating to 
the primary $\beta$-like process of phase II, can be redrawn in Fig. 8 . So, the corresponding $m$-index for phase II, according to Eq. (5) denoted as $m_{[\mathrm{II}]}$ (following the same notation as in the preceding case) would be 18 .

\section{CONCLUDING REMARKS}

The nature of glass transitions in $\mathrm{C}_{8}-\mathrm{OH}$ was studied through thermodynamic and $\mathrm{x}$-ray measurements as well as dielectric spectroscopy. Two stable solid states of $\mathrm{C}_{8}-\mathrm{OH}$, denoted as phases I and II, are known to exhibit certain degrees of disorder, each one giving rise to a glass transition when cooling down fast enough to prevent solid state phase transitions.

The stable phase I (the solid state previous to the melting), whose lattice symmetry is known to be cubic, was widely reported as an OD-state. Its dielectric loss spectra depict well developed $\alpha$-relaxation along with two $\beta$ and $\gamma$ secondary processes. If phase I is adequately cooled, the orientational disorder can be frozen-in giving rise to an OG for which the glass transition temperature was obtained by means of specific heat, x-ray, and dielectric measurements.

The stable phase II, which is stable at lower temperatures than phase I, was earlier described as an OD-state by some authors but we certainly cannot agree with this statement. As a relevant finding, we conclude that the nature of the disorder in phase II is intra-molecular and possibly due to conformational dynamic changes of the molecule as well as hydroxyl group rotations. So, phase II is proposed to be denoted as a condis-state. Once phase II is slowly cooled down to 90-100 $\mathrm{K}$, the conformational disorder would be frozen-in, giving rise to a CG. The glass transition temperature was determined through specific-heat as well as X-ray measurements. The primary $\beta$-like process of phase II could allow us to infer the glass transition temperature as well.

\section{ACKNOWLEDGMENTS}

The authors are grateful to the DGE (P98-0923 grant) and to the DURSI (SGR2002-00152 grant) for financial support.
*Electronic mail: david.orencio.lopez@upc.es

${ }^{1}$ J. Wong and C. A. Angell, Glass Structure: By Spectroscopy (Marcel Dekker, New York, 1976).

${ }^{2}$ J. Jäckl, Rep. Prog. Phys. 49, 171 (1986).

${ }^{3}$ C. A. Angell, L. E. Busse, E. I. Cooper, R. K. Kadiyala, A. Dworkin, M. Ghelfenstein, H. Szwarc, and A. Vassal, J. Chem. Phys. 82, 267 (1985).

${ }^{4}$ C. A. Angell, J. Non-Cryst. Solids 131-133, 13 (1991).

${ }^{5}$ P. W. Anderson, Science 267, 1615 (1995).

${ }^{6}$ M. D. Ediger, C. A. Angell, and S. R. Nagel, J. Phys. Chem. 100, 13200 (1996).

${ }^{7}$ K. L. Ngai, J. Non-Cryst. Solids 275, 7 (2000).

${ }^{8}$ C. A. Angell, K. L. Ngai, G. B. McKenna, P. F. McMillan, and S. W. Martin, J. Appl. Phys. 88, 3113 (2000).

${ }^{9}$ P. Lunkenheimer, U. Schneider, R. Brand, and A. Loidl, Contemp. Phys. 41, 15 (2000).

${ }^{10}$ J. Timmermans, J. Chim. Phys. Phys.-Chim. Biol. 35, 331 (1938).

${ }^{11}$ A. Michils, Bull. Soc. Chim. Belg. 57, 575 (1948).

${ }^{12}$ H. Suga and S. Seki, J. Non-Cryst. Solids 16, 171 (1974).

${ }^{13}$ U. T. Höchli, K. Knorr, and A. Loidl, Adv. Phys. 39, 405 (1990).

${ }^{14}$ B. Wunderlich and J. Grebowicz, Adv. Polym. Sci. 60/61, 1 (1984).

${ }^{15}$ B. Wunderlich, M. Möller, and H. G. Wiedemann, Mol. Cryst. Liq. Cryst. 140, 211 (1986).

${ }^{16}$ R. Edelman and A. Würflinger, Mol. Cryst. Liq. Cryst. 148, 249 (1987).

${ }^{17}$ H. Forsman and O. Andersson, J. Non-Cryst. Solids 131-133, 1145 (1991).

${ }^{18}$ J. Sciescinsky, J. Mayer, T. Wasiutinsky, E. Sciesinska, and J. Wojtowicz, Phase Transitions 54, 15 (1995).

${ }^{19}$ O. Andersson and R. G. Ross, Mol. Phys. 71, 523 (1990).

${ }^{20}$ A. Dworkin, A. H. Fuchs, M. Ghelfestein, and H. Szwarc, J. Phys. (France) Lett. 43, L21 (1982).

${ }^{21}$ M. Shablakh, L. A. Dissado, and R. M. Hill, J. Chem. Soc., Faraday Trans. 79, 383 (1983).

${ }^{22}$ A. H. Fuchs, J. Virlet, D. André, and H. Szwarc, J. Chim. Phys. Phys.-Chim. Biol. 82, 293 (1985).
${ }^{23}$ U. Poser and A. Würflinger, Ber. Bunsenges. Phys. Chem. 92, 765 (1988).

${ }^{24}$ A. Würflinger, Ber. Bunsenges. Phys. Chem. 95, 1040 (1991).

${ }^{25}$ D. L. Leslie-Pelecky and N. O. Birge, Phys. Rev. Lett. 72, 1232 (1994).

${ }^{26}$ D. L. Leslie-Pelecky and N. O. Birge, Phys. Rev. B 50, 13250 (1994).

${ }^{27}$ R. Brand, P. Lunkenheimer, and A. Loidl, Phys. Rev. B 56, R5713 (1997).

${ }^{28}$ P. Lunkenheimer, R. Brand, U. Schneider, and A. Loidl, Philos. Mag. B 79, 1945 (1999).

${ }^{29}$ M. Tyagi and S. S. N. Murthy, J. Chem. Phys. 114, 3640 (2001).

${ }^{30}$ Handbook of Chemistry and Physics, 54th ed. (CRC Press, Cleveland, 1973-1974), p. C-260.

${ }^{31}$ M. A. Rute, J. Salud, P. Negrier, D. O. López, J. Ll. Tamarit, R. Puertas, M. Barrio, and D. Mondieig, J. Phys. Chem. B 107, 5914 (2003).

${ }^{32}$ M. B. Sied, J. Salud, D. O. López, M. Barrio, and J. Ll. Tamarit, Phys. Chem. Chem. Phys. 4, 2587 (2002).

${ }^{33}$ B. Wunderlich, A. Boller, I. Okazaki, and S. Kreitmeier, Thermochim. Acta 282/283, 143 (1996).

${ }^{34}$ I. Hatta, H. Ichikawa, and M. Todoki, Thermochim. Acta 267, 83 (1995).

${ }^{35}$ B. Wunderlich, A. Boller, I. Okazaki, and K. Ishikiriyama, Thermochim. Acta 304/305, 125 (1997).

${ }^{36}$ J. C. Van Miltenburg, G. J. K. van der Berg, and M. J. van Bommel, J. Chem. Thermodyn. 19, 1129 (1987).

${ }^{37}$ J. C. Van Miltenburg, A. C. G. van Genderen, and G. J. K. van der Berg, Thermochim. Acta 319, 151 (1998).

${ }^{38}$ M. R. De la Fuente, M. A. Pérez-Jubindo, J. Zubia, T. PérezIglesias, and A. Seoane, Liq. Cryst. 16, 1051 (1994).

${ }^{39}$ C. A. Angell, J. Phys. Chem. Solids 49, 863 (1988).

${ }^{40}$ R. Böhmer and C. A. Angell, Phys. Rev. B 45, 10091 (1992).

${ }^{41}$ D. J. Plazek and K. L. Ngai, Macromolecules 24, 1222 (1991).

${ }^{42}$ R. Böhmer, K. L. Ngai, C. A. Angell, and D. J. Plazek, J. Chem. Phys. 99, 4201 (1993). 